Coagulopatía y trombosis: similitudes y diferencias entre coronavirus patogénicos

\title{
Coagulopathy and thrombosis: similarities and differences among pathogenic coronaviruses
}

https://doi.org/10.23938/ASSN.0885

\section{J.A. Páramo}

\section{RESUMEN}

La coagulopatía asociada a la enfermedad del coronavirus 2019 (COVID-19), causada por el virus SARSCoV-2, tiene implicaciones pronósticas por ser responsable de alteraciones analíticas, como el aumento de dímero D, y manifestaciones trombóticas, fundamentalmente tromboembolismo venoso, que obligan a estrategias de tromboprofilaxis. En esta revisión se revisan las principales características clínicas de la infección COVID-19 en comparación con otras infecciones por coronavirus, como el síndrome respiratorio agudo grave (SARS) y el síndrome respiratorio de Oriente Medio (MERS), con énfasis en aspectos clínicos, diagnósticos y terapéuticos.

Palabras clave. Coronavirus. Coagulación. Trombosis. Dimero D. Tromboprofilaxis.

\begin{abstract}
One of the most significant negative prognostic factors in patients suffering from the disease caused by SARS-CoV-2 (COVID-19) is the development of coagulopathy, associated with abnormal laboratory findings, such as increased D-dimer, and venous thromboembolic complications, requiring thromboprophylactic strategies. The main clinical characteristics of COVID-19 patients are revised here as compared to other coronavirus infections, such as Severe Acute Respiratory Syndrome (SARS) and Middle East Respiratory Syndrome (MERS), emphasizing clinical, diagnostic and therapeutic aspects.
\end{abstract}

Keywords. Coronaviruses. Coagulation. Thrombosis. D-dimer. Thromboprophylaxis.
Servicio de Hematología. Clínica Universidad de Navarra. Pamplona.

Instituto de Investigación Sanitaria de Navarra. CIBERCV

Recepción: 25/05/2020

Aceptación provisional: 15/07/2020

Aceptación definitiva: 21/07/20200

\section{Correspondencia:}

José A. Páramo

Servicio de Hematología

Clínica Universidad de Navarra

Avda. Pío XII, 36

31008 Pamplona, España

E-mail: japaramo@unav.es 


\section{IMPLICACIONES DE LA INFECCIÓN POR CORONAVIRUS EN LOS PROCESOS TROMBÓTICOS}

Aproximadamente uno de cada cinco pacientes infectados por el coronavirus SARS-CoV-2 desarrolla una infección grave, caracterizada por neumonía, sepsis, síndrome respiratorio agudo y coagulacion intravascular diseminada (CID), cuyo curso puede resultar fatal ${ }^{1-3}$. Al proceso patológico que causa esta nueva infección se le ha denominado enfermedad del coronavirus 2019 o COVID-19.

Asimismo, se ha puesto de manifiesto que los pacientes hospitalizados que presentan manifestaciones respiratorias o sistémicas graves muestran una coagulopatía que se asocia con complicaciones trombóticas, fundamentalmente tromboembolismo venoso (TEV), en hasta el $25-30 \%$ de los $\operatorname{casos}^{4,5}$. Dicha coagulopatía es multifactorial, ya que están alterados los tiempos de coagulación y la cifra de plaquetas; también se observa un marcado aumento de dímero $\mathrm{D}$, un producto de degradación de la fibrina, reflejando una activación del mecanismo de coagulación y fibrinolisis. Un pequeño porcentaje de pacientes desarrollan el cuadro de CID, caracterizada por consumo de plaquetas, factores de coagulación, aumento de fibrinolisis y microtrombosis generalizada asociada con fallo multiorgánico y elevada mortalidad ${ }^{6,7}$.

Si bien el mecanismo de la coagulopatía no se ha establecido con precisión, es conocido que las infecciones virales inducen una respuesta inflamatoria sistémica acompañada de una tormenta de citocinas, que provoca una alteración del equilibrio entre los mecanismos pro y anticoagulantes, y ello conlleva disfunción endotelial, elevación del factor von Willebrand y de factor tisular, estimulación plaquetar y alteraciones de la fibrinolisis, que culmina con la generación de fibrina a nivel vascular. A su vez, la hipoxemia induce liberación de factores inducibles por hipoxia (HIF) que también promueven la activación de la coagulación y disminución de las fibrinolisis, contribuyendo, asimismo, a la generación de fibrina y favoreciendo la trombosis. Finalmente, las plaquetas actuarían como sensores que, tras reconocer antígenos virales, interaccionarían con los leucocitos favoreciendo su activación, lo que se ha denominado inmunotrombosis ${ }^{8-12}$. Por consiguiente, las interacciones entre macrófagos, monocitos, células endoteliales, plaquetas y linfocitos juegan un papel determinante en el efecto procoagulante de las infecciones víricas.

Sin embargo, estas manifestaciones no son específicas del SARS-CoV-2, ya que también han estado presentes en otras infecciones por coronavirus ocurridas en los últimos 15 años, como el síndrome respiratorio agudo grave (SARS) y el síndrome respiratorio de Oriente Medio (MERS), donde se producen numerosas alteraciones de la coagulación pero con algunas diferencias respecto a la COVID-19 ${ }^{13,14}$ (Tabla 1$)$.

Tabla 1. Diferencias en coagulopatía y trombosis entre COVID-19 y otras infecciones causadas por coronavirus

\begin{tabular}{lcc}
\hline & COVID-19 & SARS y MERS \\
\hline CID & +++ & ++ \\
Embolismo pulmonar & +++ & ++ \\
Prolongación TP & ++ & + \\
Prolongación TTPA & + & ++ \\
Aumento fibrinógeno & +++ & ++ \\
Trombocitopenia & + & ++ \\
Aumento dímero D & ++++ & ++ \\
\hline
\end{tabular}

COVID-19: enfermedad causada por SARS-CoV-2; SARS: síndrome respiratorio agudo grave, causado por SARS-CoV-1; MERS: síndrome respiratorio de Oriente Medio causado por MERS-CoV; CID: coagulación intravascular diseminada; TP: tiempo de protrombina; TTPA: tiempo de tromboplastina parcial activada. 


\section{Coagulopatía e infección por SARS-CoV-2}

La infección por SARS-CoV-2, que causa un síndrome respiratorio agudo similar a otros SARS, se inició en Wuhan (China) en 2019 y cursa con síntomas leves o moderados en más del $80 \%$ de los casos y con cuadro severo en el $16 \%$ de los mismos ${ }^{1-3}$; las alteraciones de coagulación y complicaciones trombóticas son frecuentes en estos pacientes. En una serie retrospectiva incluyendo 183 pacientes con síntomas graves, Tang y col describieron que un $71.4 \%$ de los pacientes fallecidos cumplían criterios de CID, frente al $0,6 \%$ de los supervivientes ${ }^{6}$. En esta y otras series se describieron alteraciones de las pruebas de coagulación, incluyendo aumento del dímero D (36-50\%), prolongación del tiempo de protrombina (30\%) y de tromboplastina parcial activado (TTPA) (16\%), y trombocitopenia (20-30\%). Sin embargo, solo un $6,4 \%$ de los pacientes que fallecieron desarrollaron CID. Estos estudios fueron la base para la administración de tromboprofilaxis con heparina de bajo peso molecular (HBPM) o heparina no fraccionada en los sujetos que cumplían los criterios de sepsis asociada a coagulopatía, o niveles de dímero D seis veces superiores a la normalidad. El empleo de tromboprofilaxis con HBPM resultó en una reducción de la mortalidad en los pacientes con coagulopatía asociada a sepsis $(64,2 \%$ sin HBPM $v$ s $40 \%$ con HBPM). La tromboprofilaxis también fue beneficiosa en la reducción de eventos trombóticos en aquellos pacientes con valores de dímero $\mathrm{D}$ seis veces superiores al valor normal $(32,8 \%$ con HBPM vs $52,5 \%$ sin HBPM) $^{15}$.

Un estudio prospectivo reciente en 150 pacientes ingresados en UCI con síndrome de distress respiratorio agudo asociado a COVID-19 describió complicaciones trombóticas en 64 pacientes (42,6\%) durante su estancia en UCI, con predomino de embolismo pulmonar en 25 pacientes (16,7\%) que se presentó a los 5,5 días tras la admisión en la unidad. Dos pacientes presentaron ictus isquémico en territorio cerebeloso y un paciente isquemia mesentérica. En relación a los parámetros de coagulación, la mayoría de los pacientes ( $>95 \%)$ presentaron elevación de dímero Dy de fibrinógeno y, en menor pro- porción, un alargamiento del tiempo de protrombina (28\%) y trombocitopenia (20\%)

\section{Coagulopatía e infección por SARS-CoV-1}

La epidemia por coronavirus SARSCoV-1 surgió en 2003 en Guandong (China) y se extendió por numerosos países. De forma similar a la COVID-19, también se ha asociado con complicaciones trombóticas y alteraciones de la coagulación. Así, en estudios necrópsicos se han detectado a nivel histológico microtrombos de fibrina en la circulación pulmonar, bronquial y en pequeños vasos, indicando que el efecto protrombótico del virus se localiza preferentemente a nivel pulmonar $^{16}$. Otros trabajos similares han confirmado, en casos aislados, la presencia de trombosis en la circulación pulmonar, tromboembolismo venoso, ictus isquémico y trombosis microvascular ${ }^{17-20}$.

A nivel de laboratorio se ha descrito alargamiento del tiempo de protrombina y del TTPA, aumento de dímero D y trombocitopenia. En comparación con la COVID-19, se han observado mayores tasas de trombocitopenia (hasta $50 \%$ ) y prolongación del TTPA (40-60\%), y valores similares o algo inferiores de dímero $\mathrm{D}^{21,22}$. La presencia de trombocitopenia en combinación con mialgia, fiebre, rinorrea y linfopenia detectó infección por SARS-CoV-1 con $100 \%$ de sensibilidad y $86,5 \%$ de especificidad ${ }^{22}$. Sin embargo, a diferencia de la COVID-19, las alteraciones de la coagulación no se relacionaron con una mayor proporción de ingreso en UCI o superior mortalidad ${ }^{23}$.

Se ha estudiado in vitro el efecto de este virus en células mononucleares de sangre periférica, observándose un aumento en la expresión de genes procoagulantes, incluyendo fibrinógeno, serpina A3, factor II, factor tisular y factor X. Un análisis similar en células de hepatoma (Huh7) reveló sobreexpresión de diversos genes protrombóticos, como el inhibidor de la vía del factor tisular (TFPI2), inhibidor del activador tisular del plasminógeno (PAI-1) y trombospondina. Finalmente, se observó alteración de la fibrinolisis por disregulación de urocinasa, un activador del plasminógeno ${ }^{24,25}$. 


\section{Coagulopatía e infección por MERS-CoV}

En 2012 se identificó otro coronavirus en Arabia Saudí, denominado MERS-CoV, responsable del denominado síndrome respiratorio de Oriente Medio. Al igual que los otros coronavirus, se asoció con complicaciones trombóticas y alteraciones de la coagulación, si bien los estudios realizados sobre el papel de la coagulación son escasos comparados con las otras epidemias ${ }^{26,27}$.

En pacientes con infección por MERSCoV se observó una disminución de la cifra de plaquetas en el $36 \%$ de los casos, aunque los valores encontrados estaban dentro de límites normales y no existían diferencias en relación a la evolución moderada o severa de la enfermedad. También se describieron casos aislados de CID y fallo multiorgánico. Sin embargo, muy pocos pacientes presentaron trombocitopenia o coagulopatía ${ }^{28}$. En la serie de Saad y col la tasa de mortalidad fue del $60 \%$; la edad superior a 65 años y la existencia de infecciones concomitantes, pero no las alteraciones hemostáticas, fueron las variables que se asociaron significativamente con la mortalidad ${ }^{29}$.

Respecto a estudios analíticos, se demostró a nivel experimental que los ratones transgénicos que expresaban la proteína dipeptidil peptidasa 4 (hDPP4, considerada la proteína receptora que facilita la entrada del virus en las células) desarrollaron fallo multiorgánico y manifestaciones neurológicas; a nivel histológico se observaron microtrombos en la vasculatura pulmonar a partir del cuarto día de infección, coincidiendo con zonas de consolidación pulmonar y edema alveolar ${ }^{30}$. Estos hallazgos indican que MERS es capaz de activar la coagulación e inducir la formación de microtrombos.

\section{CONCLUSIONES}

La alteración de los mecanismos de coagulación y formación de fibrina a nivel pulmonar y sistémico es un hallazgo frecuente en todas las infecciones por coronavirus asociadas con enfermedad pulmonar grave y con una respuesta inflamatoria sistémica, tanto en clínica humana como en modelos animales, si bien existen algunas diferencias entre ellas. Estos factores contribuyen a la alta prevalencia de complicaciones trombóticas asociadas con estas infecciones víricas, que tienen un gran impacto pronóstico y requieren estrategias de tromboprofilaxis en los pacientes.

\section{BIBLIOGRAFÍA}

1. Zhu N, Zhang D, Wang W, Li X, Yang B, Song J et al. Novel Coronavirus Investigating and Research Team. A novel coronavirus from patients with pneumonia in China, 2019. N Engl J Med 2020; 382: 727-733.

2. Huang C, Wang Y, Li X, Ren L, Zhao J, Hu Y et al. Clinical features of patients infected with 2019 novel coronavirus in Wuhan, China. Lancet 2020; 395: 497-506.

3. Guan WJ, Ni ZY, Hu Y, Liang WH, Ou CQ, He JX et al. Treatment Expert Group for Covid-19. Clinical characteristics of coronavirus disease 2019 in China. N Engl J Med 2020; 382: 1708-1720.

4. Helms J, Tacquard C, Severac F, Leonard-Lorant I, Ohana M, Delabranche X et al. High risk of thrombosis in severe SARS-CoV-2 infection: a multicenter prospective cohort study. Intensive Care Med 2020; 46: 1089-1098. https:// doi.org/10.1007/s00134-020-06062-x

5. Bikdeli B, Madhavan MV, Jimenez D, Chuich T, DreYFus I, DRIGgin E et al. COVID-19 and thrombotic or thromboembolic disease: implications for prevention, antithrombotic therapy, and follow-up. J Am Coll Cardiol 2020; 23: 29502973.

6. TANG N, Li D, WANG X, Sun Z. Abnormal coagulation parameters are associated with poor prognosis in patients with novel coronavirus pneumonia. J Thromb Haemost 2020; 18: 844847. https://doi.org/10.1111/jth.14768

7. Henry BM, de Oliveira MHS, Benoit S, PlebaNI M, LIPPI G. Hematologic, biochemical and immune biomarker abnormalities associated with severe illness and mortality in coronavirus disease 2019 (COVID-19): a metaanalysis. Clin Chem Lab Med 2020. https:// doi.org/10.1515/cclm-2020-0369

8. Gralinski LE, Bankhead A III, Jeng S, Menachery VD, Proll S, Beliste SE et al. Mechanisms of severe acute respiratory syndrome coronavirus-induced acute lung injury. mBio 2013; 4. https://doi.org/10.1128/mbio.00271-13

9. AntoniaK S, Mackman N. Multiple roles of the coagulation protease cascade during virus 
infection. Blood 2014; 123: 2605-2613. https:// doi.org/10.1182/blood-2013-09-526277

10. Subramaniam S, Scharrer I. Procoagulant activity during viral infections. Front Biosci 2018; 23: 1060-1081. https://doi.org/10.2741/4633

11. Gupta N, Zhao YY, Evans CE. The stimulation of thrombosis by hypoxia. Thromb Res 2019; 181: 77-83.

12. PÁramo JA. Neutrophils as instigators of thrombosis: beyond antimicrobial protection. Rev Clin Esp 2020. https://doi. org/10.1016/j.rce.2019.11.01

13. Giannis D, Ziogas IA, Gianni P. Coagulation disorders in coronavirus infected patients: $\mathrm{CO}$ VID-19, SARS-CoV-1, MERS-CoV and lessons from the past. J Clin Virol 2020; 127: 104362. https://doi.org/10.1016/j.jcv.2020.104362

14. Yin S, Huang M, Li D, TANG N. Difference of coagulation features between severe pneumonia induced by SARS-CoV2 and non-SARS-CoV2. J Thromb Thrombolysis 2020. https://doi. org/10.1007/s11239-020-02105-8

15. TANG N, Bai H, Chen X, Gong J, Li D, Sun Z. Anticoagulant treatment is associated with decreased mortality in severe coronavirus disease 2019 patients with coagulopathy. J Thromb Haemost 2020; 18: 1094-1099. https://doi.org/10.1111/jth.14817

16. Nicholls JM, Poon LL, LeE KC, NG WF, Lai ST, LEUNG CY et al. Lung pathology of fatal severe acute respiratory syndrome. Lancet 2003; 361: 1773-1778.

17. Chong PY, Chui P, Ling AE, Franks TJ, Tai DY, Leo YS et al. Analysis of deaths during the severe acute respiratory syndrome (SARS) epidemic in Singapore: challenges in determining a SARS diagnosis. Arch Pathol Lab Med 2004; 128: 195-204.

18. Umapathi T, Kor AC, Venketasubramanian N, Lim CC, PANG BC, YEO TT et al. Large artery ischaemic stroke in severe acute respiratory syndrome (SARS). J Neurol 2004; 251: 1227-1231. https://doi.org/10.1007/s00415-004-0519-8

19. Ng WF, Wong SF, Lam A, MaK YF, Yao H, LeE KC et al. The placentas of patients with severe acute respiratory syndrome: a pathophysiological evaluation. Pathology 2006; 38: 210-218. https://doi.org/10.1080/00313020600696280

20. Xiang-Hua Y, Le-Min W, Ai-Bin L, Zhu G, Riquan $\mathrm{L}, \mathrm{Xu}-\mathrm{You} \mathrm{Z}$ et al. Severe acute respiratory syndrome and venous thromboembolism in multiple organs. Am J Respir Crit Care Med 2010; 182: 436-437. https://doi.org/10.1164/ ajrccm.182.3.436

21. Wong RS, Wu A, To KF, Lee N, Lam CW, Wong $\mathrm{CK}$ et al. Haematological manifestations in patients with severe acute respiratory syndrome: retrospective analysis. BMJ 2003; 326: 1358-1362. https://doi.org/10.1136/ bmj.326.7403.1358

22. Chen SY, Su CP, Ma MH, Chiang WC, Hsu CY, $\mathrm{Ko} \mathrm{PC}$ et al. Predictive model of diagnosing probable cases of severe acute respiratory syndrome in febrile patients with exposure risk. Ann Emerg Med 2004; 43: 1-5. https:// doi.org/10.1016/s0196-0644(03)00817-5

23. Lee N, Hui D, Wu A, Chan P, Cameron P, Joynt GM et al. A major outbreak of severe acute respiratory syndrome in Hong Kong. N Engl J Med 2003; 348: 986-994.

24. Ng LF, Hibberd ML, Ooi EE, TAng KF, Neo SY, TAN J et al. A human in vitro model system for investigating genome-wide host responses to SARS coronavirus infection. BMC Infect Dis 2004; 4: 34. https://doi.org/10.1186/14712334-4-34

25. GRALINSKI LE, BARIC RS. Molecular pathology of emerging coronavirus infections. J Pathol 2015; 235: 185-195. https://doi.org/10.1002/ path. 4454

26. Assiri A, Al-Tawfig JA, Al-Rabeeah AA, Al-Rabiah FA, Al-HajJar S, Al-BarraK A et al. Epidemiological, demographic, and clinical characteristics of 47 cases of Middle East respiratory syndrome coronavirus disease from Saudi Arabia: a descriptive study. Lancet Infect Dis 2013; 13: 752-761. https://doi.org/10.1016/ s1473-3099(13)70204-4

27. Hwang SM, Na BJ, Jung Y, Lim HS, SEo JE, Park SA et al. Clinical and laboratory findings of Middle East respiratory syndrome coronavirus infection. Jpn J Infect Dis 2019; 72: 160-167. https://doi.org/10.7883/yoken. jjid. 2018.187

28. SINGH SK. Middle East respiratory syndrome virus pathogenesis. Semin Respir Crit Care Med 2016; 37: 572-577. https://doi. org/10.1055/s-0036-1584796

29. SAad M, Omrani AS, Baig K, Bahloul A, Elzein F, MATin MA et al. Clinical aspects and outcomes of 70 patients with Middle East respiratory syndrome coronavirus infection: a single-center experience in Saudi Arabia. Int J Infect Dis 2014; 29: 301-306. https://doi. org/10.1016/j.ijid.2014.09.003

30. Li K, Wohlford-Lenane C, Perlman S, Zhao J, JEWELL AK, REZNIKOV LR et al. Middle east respiratory syndrome coronavirus causes multiple organ damage and lethal disease in mice transgenic for human dipeptidyl peptidase 4. J Infect Dis 2016; 213: 712-722. https://doi. org/10.1093/infdis/jiv499 
